\title{
ON IDENTIFIABILITY OF LINEAR INFINITE-DIMENSIONAL SYSTEMS
}

\author{
Yury Orlov \\ Electr. Dept., CICESE Research Center \\ Km. 107, Carretera Tijuana-Ensenada, Ensenada, B.C., Mexico 22860 \\ yorlov@cicese.mx
}

\begin{abstract}
Identifiability analysis is developed for linear dynamic systems evolving in a Hilbert space. Finite-dimensional sensing and actuation are assumed to be only available. Identifiability conditions for the transfer function of such a system is constructively addressed in terms of sufficiently nonsmooth controlled inputs. The introduced notion of a sufficiently nonsmooth input does not relate to a system and it can therefore be verified independently of any particular underlying system.
\end{abstract}

Keywords: Identifiability, Hilbert space, Markov parameters, sufficiently rich input.

\section{Introduction}

A standard approach to identifying a linear system implies that the structure of the system is deduced by using physical laws and the problem is in finding the values of parameters in the state equation. The ability to ensure this objective is typically referred to as parameter identifiability. For complex systems, however, it may not be possible to model all of the system and a black box approach should be brought into play. Here knowledge of the input-output map comes from controlled experiments. The questions then arise as to if the input-output map and the transfer function of the system have a one-to-one relation and in which sense a state space model, if any, is unique.

Many aspects of the identifiability of linear finite-dimensional systems are now well-understood. In this regard, we refer to [1], [5], and [7] to name a few monographs.

Recently [8], [9], the identifiability analysis was proposed in an infinitedimensional setting for linear time-delay systems with delayed states, control inputs and measured outputs, all with a finite number of lumped delays. It was demonstrated that the transfer function of such a system 
can be re-constructed on-line whenever a sufficiently nonsmooth input signal is applied to the system. The present work extends this result to linear dynamic systems evolving in a Hilbert space. The transfer function identifiability conditions for these systems are also addressed in terms of sufficiently nonsmooth input signals which are constructively introduced in the Hilbert space. The notion of a sufficiently nonsmooth input does not relate to a system and it can therefore be verified independently of any particular underlying system.

Similar to linear finite-dimensional systems, the parameter identifiability in a Hilbert space requires the system with unknown parameters to be specified in a form such that if confined to this form all the unknown parameters are uniquely determined by the transfer function. In contrast to [2], [3], and [10] where the parameter identifiability is established for linear distributed parameter systems with sensing and actuation distributed over the entire state space, the present development deals with a practical situation where finite-dimensional sensing and actuation are only available.

\section{Basic Definitions}

We shall study linear infinite-dimensional systems

$$
\begin{aligned}
& \dot{x}=A x+B u, x(0)=x^{0} \\
& y=C x,
\end{aligned}
$$

defined in a Hilbert space $H$, where $x \in H$ is the state, $x^{0}$ is the initial condition, $u \in R^{m}$ is the control input, $y \in R^{p}$ is the measured output, $A$ is an infinitesimal operator with a dense domain $\mathcal{D}(A), B$ is the input operator, $C$ is the measurement operator. All relevant background materials on infinite-dimensional dynamic systems in a Hilbert space can be found, e.g., in [4].

The following assumptions are made throughout:

$1 A$ generates an analytical semigroup $S_{A}(t)$ and has compact resolvent;

$2 B \in \mathcal{L}\left(R^{m}, H\right)$ and $C \in \mathcal{L}\left(H, R^{p}\right)$;

$3 x(0)=x^{0} \in \bigcap_{n=1}^{\infty} \mathcal{D}\left(A^{n}\right)$;

$4 \mathcal{R}(B) \in \bigcap_{n=1}^{\infty} \mathcal{D}\left(A^{n}\right)$.

Hereafter, the notation is fairly standard. The symbol $\mathcal{L}(U, H)$ stands for the set of linear bounded operators from a Hilbert space $U$ to $H$; $\mathcal{D}(A)$ is for the domain of the operator $A ; \mathcal{R}(B)$ denotes the range of the operator $B$. 
The above assumptions are made for technical reasons. It is wellknown that under these assumptions, the Hilbert space-valued dynamic system (1), driven by a locally integrable input $u(t)$, has a unique strong solution $x(t)$, globally defined for all $t \geq 0$. The spectrum $\sigma(A)=$ $\left\{\lambda_{n}\right\}_{n=1}^{\infty}$ of the operator $A$ is discrete, $\lambda_{n} \rightarrow-\infty$ as $n \rightarrow \infty$, and the solution of (1) can be represented in the form of the Fourier series

$$
x(t)=\Sigma_{n=1}^{\infty}\left\{\prec x^{0}, r_{n} \succ e^{\lambda_{n} t}+\int_{0}^{t} \prec B u(\tau), r_{n} \succ e^{\lambda_{n}(t-\tau)} d \tau\right\} r_{n}
$$

written in terms of the eigenvectors $r_{n}, n=1, \ldots$ of the operator $A$ and the inner product $\prec,, \succ \succ$ in the Hilbert space $H$. Furthermore, this solution is regular enough in the sense that $x(t) \in \bigcap_{n=1}^{\infty} \mathcal{D}\left(A^{n}\right)$ for all $t \geq 0$.

The identifiability concept is based on the comparison of system (1), (2) and its reference model

$$
\begin{array}{r}
\dot{\hat{x}}(t)=\hat{A} \hat{x}+\hat{B} u, \hat{x}(0)=\hat{x}^{0} \\
\hat{y}(t)=\hat{C} \hat{x}
\end{array}
$$

defined on a Hilbert space $\hat{H}$ with the initial condition $\hat{x}^{0}$ and operators $\hat{A}, \hat{B}, \hat{C}$, substituted in (1), (2) for $x^{0}$ and $A, B, C$, respectively. Certainly, Assumptions 1-4 remain in force for the reference model (4), (5).

The transfer function $T(\lambda)=C(\lambda I-A)^{-1} B$ of system (1), (2) is completely determined by means of the Markov parameters $C A^{n-1} B, n=$ $1,2, \ldots$ through expanding into the Laurent series

$$
T(\lambda)=\Sigma_{n=1}^{\infty} \lambda^{-n} C A^{n-1} B,
$$

and the identifiability of the transfer function is introduced as follows.

DEFINITION 1 The transfer function of (1), (2), or equivalently, the Markov parameters of system (1), (2) are said to be identifiable on $\bigcap_{n=1}^{\infty} \mathcal{D}\left(A^{n}\right)$ iff there exists a locally integrable input function $u(t)$ to be sufficiently rich for the system in the sense that the identity $y(t) \equiv \hat{y}(t)$ implies that

$$
C A^{n-1} B=\hat{C} \hat{A}^{n-1} \hat{B}, n=1,2, \ldots
$$

(and consequently $T(\lambda)=\hat{T}(\lambda)$ ), regardless of a choice of the initial conditions $x^{0} \in \bigcap_{n=1}^{\infty} \mathcal{D}\left(A^{n}\right), \hat{x}^{0} \in \bigcap_{n=1}^{\infty} \mathcal{D}\left(\hat{A}^{n}\right)$. In that case the identifiability is said to be enforced by the input $u(t)$.

The identifiability of the system itself (rather than its transfer function) is addressed in a similar manner. 
Definition 2 System (1), (2) is said to be identifiable on $\bigcap_{n=1}^{\infty} \mathcal{D}\left(A^{n}\right)$ iff there exists a locally integrable input function $u(t)$ such that the identity $y(t) \equiv \hat{y}(t)$ implies that

$$
C=\hat{C}, A=\hat{A}, B=\hat{B},
$$

regardless of a choice of the initial conditions $x^{0} \in \bigcap_{n=1}^{\infty} \mathcal{D}\left(A^{n}\right), \hat{x}^{0} \in$ $\bigcap_{n=1}^{\infty} \mathcal{D}\left(\hat{A}^{n}\right)$.

For later use, we also define sufficiently nonsmooth inputs, which form an appropriate subset of sufficiently rich inputs. Indeed, while being applied to a finite-dimensional system, a nonsmooth input has enough frequencies in the corresponding Fourier series representation and hence it turns out to be sufficiently rich in the conventional sense [6].

Let $u(t)=\sum_{i=1}^{m} u_{i}(t) e_{i}$ be a piece-wise smooth input, expanded in the basis vectors $e_{i} \in R^{m}, i=1, \ldots m$, and let $D_{i}$ be the set of discontinuity points $t \geq 0$ of $u_{i}(t)$.

Definition 3 The input $u(t)=\Sigma_{i=1}^{m} u_{i}(t) e_{i}$ is said to be sufficiently discontinuous iff for any $i=1, \ldots, m$ there exists $t_{i} \in D_{i}$ such that $t_{i} \notin D_{j}$ for all $j \neq i$.

DEFINITION 4 The input $u(t)=\sum_{i=1}^{m} u_{i}(t) e_{i}$ is said to be sufficiently nonsmooth of class $C^{l}$ iff there exists $l$-th order derivative of the input $u(t)$ and $u^{(l)}(t)$ is sufficiently discontinuous.

It is worth noticing that the notion of a sufficiently nonsmooth (discontinuous) input does not relate to a system and it can therefore be verified independently of any particular underlying system.

\section{Identifiability Analysis}

Once the infinite-dimensional system (1), (2) is enforced by a sufficiently nonsmooth input, its transfer function is unambiguously determined by the input-output map. The transfer function identifiability in that case is guaranteed by the following result.

THEOREM 5 Consider a Hilbert space-valued system (1), (2) with the assumptions above. Then the Markov parameters $C A^{n-1} B, n=1,2, \ldots$ of (1), (2) are identifiable and their identifiability can be enforced by an arbitrary sufficiently nonsmooth (particularly, sufficiently discontinuous) input $u(t)=\sum_{i=1}^{m} u_{i}(t) e_{i}$.

Proof: For certainty, we assume that the input $u(t)$ is sufficiently discontinuous. The general proof in the case where $u(t)$ is sufficiently nonsmooth is nearly the same and it is therefore omitted. 
According to Definition 1, we need to prove that the output identity

$$
C x(t) \equiv \hat{C} \hat{x}(t)
$$

implies the equivalence (6) of the Markov parameters.

By differentiating (8) along the solutions of (1) and (4), we obtain that

$$
C A x(t)+C B \Sigma_{i=1}^{m} u_{i}(t) e_{i} \equiv \hat{C} \hat{A} \hat{x}(t)+\hat{C} \hat{B} \Sigma_{i=1}^{m} u_{i}(t) e_{i} .
$$

It follows that

$$
C B=\hat{C} \hat{B}
$$

because otherwise identity (9) could not remain true at the discontinuity instants $t_{i}, i=1, \ldots, m$. Indeed, in spite of the discontinuous behavior of the input $u(t)$, the solution (3) of the differential equation (1) is continuous for all $t \geq 0$. Thus, by taking into account that $u(t)$ is sufficiently discontinuous (see Definition 3), $[C B-\hat{C} \hat{B}] u_{i}(t) e_{i}$ is the only term in (9), discontinuous at $t=t_{i}$. Hence, $[C B-\hat{C} \hat{B}] e_{i}=0$ for each $i=1, \ldots, m$, thereby yielding (10).

Now (9) subject to (10) is simplified to

$$
C A x(t) \equiv \hat{C} \hat{A} \hat{x}(t)
$$

and differentiating (11) along the solutions of (1) and (4), we arrive at

$$
C A^{2} x(t)+C A B \Sigma_{i=1}^{m} u_{i}(t) e_{i} \equiv \hat{C} \hat{A}^{2} \hat{x}(t)+\hat{C} \hat{A} \hat{B} \Sigma_{i=1}^{m} u_{i}(t) e_{i} .
$$

Following the same line of reasoning as before, we conclude from (12) that

$$
C A B=\hat{C} \hat{A} \hat{B} .
$$

Finally, the required equivalence (6) of the Markov parameters for $n \geq 3$ is obtained by iterating on the differentiation. Theorem 5 is thus proven.

In a fundamental term, Theorem 5 says that the input-output map (1), (2) and Markov parameters of the Hilbert space-valued system have a one-to-one relation. In general, the identifiability of the Markov parameters does not imply the identifiability of the system. Indeed, let $Q \in \mathcal{L}(H)$ be such that $\mathcal{D}(A)$ is invariant under $Q$ and $Q^{-1} \in \mathcal{L}(H)$. Then the system

$$
\begin{aligned}
\dot{x} & =Q A Q^{-1} x+Q B u, x(0)=x^{0} \\
y & =C Q^{-1} x
\end{aligned}
$$

has the same Markov parameters $C A^{n} B, n=1,2, \ldots$. 
Thus, in analogy to the finite-dimensional case, the identifiability of the infinite-dimensional system is guaranteed if it is in a canonical form such that if confined to this form the operators $A, B, C$ are uniquely determined by the Markov parameters. The major challenge will be an explicit definition of the canonical form of a linear Hilbert space-valued system (1), (2).

\section{References}

[1] B. Astrom, K.J.and Wittenmark. Adaptive Control. Addison-Wesley, Reading, MA, 1989.

[2] J. Bentsman and Y. Orlov. Reference adaptive control of spatially varying distributed parameter systems of parabolic and hyperbolic types. Internat. $J$. Adaptive Control and Signal Processing, 15:679-696, 2001.

[3] M. Böhm, M.A. Demetriou, S. Reich, and I.G. Rosen. Model reference adaptive control of distributed parameter systems. SIAM Journal on Control and Optimization, 35:678-713, 1997.

[4] R. F. Curtain and H. J. Zwart. An Introduction to Infinite-Dimensional Linear Systems Theory. Springer-Verlag, New York, 1995.

[5] Y. D. Landau. Adaptive Control - The Model Reference Approach. Marcel Dekker, New York, 1979.

[6] R. K. Miller and A. N. Michel. An invariance theorem with applications to adaptive control. IEEE Trans. Automat. Contr., 35:744-748, 1990.

[7] K. S. Narendra and A. Annaswamy. Stable Adaptive Systems. Prentice Hall, Englewood Cliffs, NJ, 1989.

[8] Y. Orlov, L. Belkoura, J.P. Richard, and M. Dambrine. On identifiability of linear time-delay systems. IEEE Trans. Automat. Contr., 47:1319-1323, 2002.

[9] Y. Orlov, L. Belkoura, J.P. Richard, and M. Dambrine. Adaptive identification of linear time-delay systems. Internat. J. Robust and Nonlinear Control, 13:857$872,20023$.

[10] Y. Orlov and J. Bentsman. Adaptive distributed parameter systems identification with enforceable identifiability conditions and reduced spatial differentiation. IEEE Trans. Automat. Contr., 45:203-216, 2000. 